\title{
"A pre-experimental study to assess the effect of educational package on knowledge regarding haematopoietic stem cell transplantation among staff nurses working in selected hospital Bhopal, Madhya Pradesh"
}

\author{
Singh T. ${ }^{1}$, Pakhide V. ${ }^{2}$, Verma $\mathrm{M}^{3 *}$ \\ DOI: https://doi.org/10.17511/ijmrr.2021.i02.01
}

1 Tripti Singh, M Sc. Nursing, CHO, Sehore, Madhya Pradesh, India.

2 Vandana Pakhide, Assistant Professor, Pragyan College of Nursing, Bhopal, Madhya Pradesh, India.

3* Mamta Verma, Associate professor, College of Nursing, AIIMS, Bhopal, Madhya Pradesh, India.

Haematopoietic stem cell transplantation is a greater positive impact on the treatment of lifethreatening diseases. Stem cell transplantation is a lifesaving procedure for several malignant and nonmalignant life-threatening diseases, more than 40,000 stem cell transplantations are being performed annually worldwide. Methods: pre-experimental, one group pre-test post-test research design was used for conducting a study in a selected hospital of Bhopal, Madhya Pradesh. Subjects comprising of 60 staff nurses selected by non-probability purposive sampling technique, who fulfilled inclusion criteria. Results: Mean post-interventional knowledge $(27.0 \pm 6.03)$ is higher than the mean pre-interventional knowledge $(15.5 \pm 5.74)$. The mean difference between pre- interventional knowledge and post-interventional knowledge is 11.5 . The computed ' $t$ ' value $15.80,2.00, P<0.05$ shows that there is a significant difference between pre-interventional knowledge and postinterventional knowledge. There was a significant association found of demographic variables in terms of working area and years of clinical experience. Conclusion: it was concluded that the educational package was effective in enhancing the knowledge of the staff nurses. The health care professional including staff nurses also having inadequate knowledge of stem cell therapy. The skilled educative session is one of the cost-effective intervention used for educating the nurses in various aspects.

Keywords: Haematopoietic stem cell transplantation, Malignant and non-malignant disorders, Educational package.

Corresponding Author

Mamta Verma, Associate professor, College of Nursing, AIIMS, Bhopal, Madhya Pradesh, India. Email: mamta.gedam@gmail.com
How to Cite this Article

To Browse

Singh T, Pakhide V, Verma M. "A pre-experimental study to assess the effect of educational package on knowledge regarding haematopoietic stem cell transplantation among staff nurses working in selected hospital Bhopal, Madhya Pradesh". Int J Med Res Rev. 2021;9(2):58-65.

Available From

https://ijmrr.medresearch.in/index.php/ijmrr/article/ view/1270

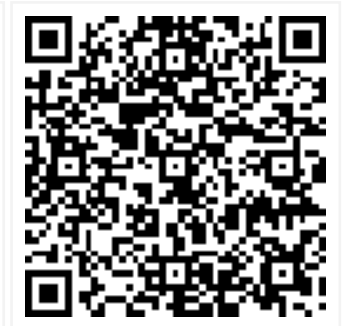

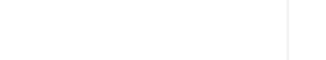




\section{Introduction}

Haematopoietic stem cell transplantation is a greater positive impact on the treatment of lifethreatening diseases. Stem cell transplantation is a lifesaving procedure for several malignant and nonmalignant life-threatening diseases, more than 40,000 stem cell transplantations are being performed annually worldwide. The stem cell is primitive cells capable of self-replication and differentiated into myeloid or lymphoid stem cells. Stem cells have the sense of capacity to divides indefinitely and have the potential to give rise to specialized cells. Haematopoietic stem cell transplantation is a useful treatment for so many conditions, such as aplastic anaemia, malignant disorders, especially myelodysplastic syndrome, leukaemia; certain types of acute leukemia, chronic and pre-leukemia states, lymphoma, multiple myeloma, neuroblastoma and selected solid tumors (breast cancer, ovarian cancer, testicular cancer, poor germ cell tumors). Non-malignant haematological disorders such as thalassemia and sickle cell anaemia, immunodeficiency diseases and wiskott-Aldrich syndrome [1].

The year 2009 marked the fiftieth anniversary of the first successful allogeneic haematopoietic stem cell transplant (HSCT). The field of HSCT has pioneered some of the most exciting areas of research today. HSCT was the original stem cell therapy, the first cancer immune therapy and the earliest example of individualized cancer therapy. In this Timeline article accomplishments made by researchers who continue to strive to improve outcomes for patients and increase the availability of this potentially lifesaving therapy for patients with otherwise incurable malignancies [2].

Hematopoietic stem cell transplantation (HSCT) is a potentially curative treatment for patients with blood disorders and genetic diseases. Approximately $70 \%$ of the HSCTs currently performed in the United States use stem cells from an unrelated donor who donated voluntarily [14]. Stem cell transplantation appears to be a promising method for the treatment of patients with neurodegenerative conditions, spinal cord injury, and stroke [15].

Multiple myeloma accounts for approximately $10 \%$ of hematologic cancers and $1 \%$ of all cancers in general. More than 30 years after its introduction, autologous stem cell transplantation (ASCT) remains the standard of care for young patients with newly diagnosed multiple myeloma [3].
As compared with single autologous stem-cell transplantation after high-dose chemotherapy, double transplantation improves overall survival among patients with myeloma, especially those who do not have a very good partial response after undergoing one transplantation [8].

Stem cell collected from different sources of the human body like bone marrow, peripheral blood and umbilical cord blood from newborn. Stem cell therapy used for tissue regeneration, developing heart tissue or any other vital tissue in the body. Leukemia patient with stem cell therapy emerge disease, stem cell therapy used for treating orthopaedic conditions, osteoarthritis, bone repair, joint repair and the neurological conditions like stroke, diabetic neuropathy and also used for antiageing therapy. Stem cell created a devastating era in medicine [4].

Stem cell transplantation (SCT) is a life-saving procedure for several malignant and non-malignant life-threatening diseases. Umbilical cord blood (UCB) which was thought to be as waste material earlier, is considered now a valuable thing. It is an important source of hematopoietic stem cells. A stem cell transplant is the infusion of healthy cells to replace diseased or damaged ones [13]. In developing countries prone to congenital malformation and genetic diseases, these are an important cause of morbidity and mortality. Due to the high birth rate, in India a very large number of the infant with genetic disorders are born with malformation and approximately 21000 with down syndrome. Nearly 80 paediatric diseases and conditions are routinely being treated with cord blood stem cells including haematopoietic and genetic disorders [5].

Autologous haematopoietic stem cell transplantation (AHSCT) is a multistep procedure that enables the destruction of the immune system and its reconstitution from haematopoietic stem cells. Originally developed for the treatment of haematological malignancies, the procedure has been adapted for the treatment of severe immunemediated disorders. Results from 20 years of research make a compelling case for selective use of AHSCT in patients with highly active multiple sclerosis (MS), and controlled trials. Immunological studies support the notion that AHSCT causes qualitative immune resetting, and have provided insight into the mechanisms that might underlie the powerful treatment effects that last well beyond recovery of immune cell numbers. 
Indeed, studies have demonstrated that AHSCT can entirely suppress MS disease activity for 4-5 years in $70-80 \%$ of patients, a rate that is higher than those achieved with any other therapies for MS. Treatment-related mortality, which was $3.6 \%$ in studies before 2005, has decreased to $0.3 \%$ in studies since 2005. Current evidence indicates that the patients who are most likely to benefit from and tolerate AHSCT are young, ambulatory and have inflammatory MS activity. Clinical trials are required to rigorously test the efficacy, safety and costeffectiveness of AHSCT against highly active MS drugs [6].

Hematopoietic stem cells (HSCs) are regarded as one of the essential cell sources for treating regenerative diseases. Among many stem cells, the feasibility of using adult-derived hematopoietic stem cells in therapeutic approaches is very diverse, and is unarguably regarded as an important cell source in stem cell biology. So far, many investigators are exploring HSCs and modified HSCs for use in clinical and basic science. In the present review, briefly summarized HSCs and their application in pathophysiologic conditions, including nonhematopoietic tissue regeneration as well as blood disorders. HSCs and HSCs-derived progenitors are promising cell sources in regenerative medicine and their contributions can be properly applied to treat pathophysiologic conditions. Among many adult stem cells, HSCs are a powerful tool to treat patients with diseases such as hematologic malignancies and liver disease. Since HSCs can be differentiated into diverse progenitors including endothelial progenitors, they may be useful for constructing strategies for effective therapy [7]. The field of hematopoietic stem cell transplant (HSCT) has made ground-breaking progress in the treatment of many malignant and non-malignant conditions. It has also pioneered the concepts of stem cell therapy and immunotherapy as a tool against cancer. The success of transplant for hematologic malignancies derives both from the ability to treat patients with intensive chemoradiotherapy and from potent graft-versusleukaemia (GVL) effects mediated by donor immunity. Additionally, HSCT has been a curative therapy for several non-malignant hematologic disorders through the provision of donor-derived haematopoiesis and immunity. Preclinical and clinical research in the field has contributed to an advanced understanding of histocompatibility, graftversus-host disease (GVHD), GVL effect, and immune reconstitution after transplant.
Improved donor selection, tailored conditioning regimens, and better supportive care have helped reduce transplant-related morbidity and mortality and expanded access. The development of unrelated donor registries and increased utilization of cord blood and partially matched related donor transplants have ensured a donor for essentially everyone who needs a transplant. However, significant barriers remain in the form of disease relapse, GVHD infectious complications, and regimen-related toxicities. Recent developments in the field of cellular therapy are expected to further improve the efficacy of transplant [9].

Allogeneic hematopoietic stem cell transplantation (allo-HSCT) from a geno-identical matched sibling (MSD) is one of the most successful therapies in patients with non-malignant haematological disorders. This study included 273 patients with severe aplastic anemia (SAA), 152 patients with BThalassemia major (BTM), 31 patients with Fanconi's anemia (FA), 20 patients with congenital immunodeficiency diseases (ID), and 13 patients with inherited metabolic disorders (IMD) allografted from an MSD. In SAA, the 8-year overall survival (OS) of the whole group of patients was $74 \%$. Allogeneic -HSCT provides a higher DFS (DiseaseFree Survival) rate over conventional therapies for patients with non-malignant haematological disorders with prolonged survival [10].

Problem Statement: "A pre-experimental study to assess the effect of educational package on knowledge regarding haematopoietic stem cell transplantation among staff nurses working in selected hospital Bhopal, Madhya Pradesh".

\section{Objectives}

- Assess the pre-interventional knowledge regarding haematopoietic stem cell transplantation among staff nurses.

- Assess the post-interventional knowledge regarding haematopoietic stem cell transplantation among staff nurses.

- Find out the significant difference between preinterventional and post-interventional knowledge regarding haematopoietic stem cell transplantation among staff nurses.

- Associate the pre-interventional knowledge scores findings regarding haematopoietic stem cell transplantation among staff nurses with their selected demographic variables. 


\section{Research Methodology}

Research methodology indicates the general pattern of organizing the procedure for gathering valid and reliable data for investigation. In this study, a quantitative research approach was adopted. Knowledge assessed by self-structured questionnaire regarding haematopoietic stem cell transplantation.

Research Design: In this study, a preexperimental one group pre-test post-test design was used to assess the effectiveness of an educational package containing literature related to haematopoietic stem cell transplantation.

Setting: This study is being conducted at Chirayu Medical College \& Hospital Bhopal.

Duration: May - June, 2019

Sample size: The study subjects were 60 staff nurses, selected through Non- probability purposive sampling technique.

Ethical Approval: A written permission was obtained to conduct the study from the administrative authorities of the institution.

\section{Inclusion criteria}

- Staff nurses who willing to participate in this study.

- Staff nurses aged between 20-50 years of age and above.

- Staff nurses who were present at the time of data collection.

\section{Exclusion criteria}

- Staff nurses who underwent a special training program related to hematopoietic stem cell transplantation.

- Staff nurses having postgraduate qualification.

\section{Development of the tool}

- Developed educational package (informational booklet and PowerPoint presentation) containing literature related to sources, collection, indications, types, histocompatibility testing, procedure, nursing management and complications related to hematopoietic stem cell transplantation.

- After content validity and modification of the tool, a structured knowledge questionnaire about the hematopoietic stem cell transplantation was developed.
Split Half method, Karl Pearson's coefficient correlation used to calculate reliability. Reliability was found $r=0.79$ which reveals tools was reliable for the study. A structured questionnaire consists of 42 multiple choice questions with four options, each multiple-choice questions had one correct answer and three wrong distracters.

- Scoring criteria, 1 (one) mark for correct answer and 0 (zero) for the wrong answer. Categorization of score done, rating ( $\left.\begin{array}{ll}0 & -14\end{array}\right)$ poor, (15 - 28) average, and (29 - 42) consider good.

Data analysis procedure - The data obtained from 60 respondents were analysed by using descriptive and inferential statistics method after consultation of the statistician, as follows:

- A master data sheet was prepared and compiled demographic data, containing selected sample characteristics was analyzed using frequency and percentage distribution.

- Data was collected and presented in the forms of tables and bar diagrams.

- Find out the mean, median and standard deviation of pre-interventional and postinterventional knowledge scores.

- ' $t$ ' test is done to find out the significant difference between pre-interventional and postinterventional scores.

- A Chi-square test was used to find out the association of pre-interventional knowledge scores with their selected demographic variables.

\section{Results}

Results in terms of the objectives of the study:

\section{- Assess the pre-interventional knowledge regarding haematopoietic stem cell transplantation among staff nurses.}

Most of the sample $67 \%$ were categorized in the poor category, $28 \%$ were in the average category and only $5 \%$ were in a good category.

Table No.1: Representing frequency and percentage distribution of pre-interventional knowledge score.

\begin{tabular}{|l|l|l|}
\hline Pre-interventional scores & Frequency $(\mathrm{N})$ & Percentage (\%) \\
\hline $0-14$ & 40 & 67 \\
\hline $15-28$ & 17 & 28 \\
\hline $29-42$ & 03 & 5 \\
\hline
\end{tabular}


(9.5 \pm 1.46$)$. The computed paired ' $t$ ' value (18.12) was found significant but there was no association of demographic variables. It means the study was effective in enhancing the knowledge of staff nurses [11].

Amidst the recent developments, stem cell therapy is going to be one of the most promising ones to treat many genetic diseases as well as organ failures. The stem cells achieved their importance as a source of treating many genetic diseases just because of their totipotent nature. Totipotency is the ability of any cell to become the choice of cell that wants to be developed. A recent study shows that almost 75 genetic diseases and more than 20, 000 transplantations could be achieved by using stem cells. The present study has been conducted among the final year B. Sc., Nursing students at P.S College of Nursing, Kanyakumari, Tamil Nadu, India. Quantitative approach and quasi-experimental were followed by the students, where one group pre-test, post-test design was adopted. Forty students were selected by the convenient sampling method. A structured questionnaire was used to assess knowledge. In a structured teaching programme, a post-test was conducted by the seventh day.

According to the pre-test results, no one had adequate knowledge, whereas $40 \%$ had inadequate knowledge and 60 per cent of the population had moderate knowledge. From the post-test analysis it is evident that, $55 \%$ of the students were aware of stem cell therapy and 40 per cent of them had moderate knowledge. The percentage of the population with inadequate knowledge brought down to a level of $5 \%$. The results reveal the fact that the structural teaching programme on stem cell therapy was very effective [12].

Yesikar, V., Banseria, R., Dixit, S., \& Shivram, G. conducted a cross-sectional study on 80 undergraduate medical students \& 80 non-medical students and 40 Antenatal mothers. The study tool for data collection in the form of a Structured Questionnaire was designed. The study was conducted for two months. Data were analysed in SPSS version 20, Chi-Square test was used to assess the significance of the responses and a Pvalue $<0.05$ was considered statistically significant. Results: $45 \%$ of medicos and $31.25 \%$ non-medicos were aware of the terminology stem cell while only $10 \%$ of Antenatal mothers knew about it. Monetary Reason was the major factor that prevented $77.5 \%$ of mothers from opting for stem cell preservation while $12.5 \%$ of Antenatal mothers never thought of
It, $7.5 \%$ of Antenatal mothers did not opt because of social stigma and $2.5 \%$ due to Ethical consideration [13].

Neha Patyal, Ramanpreet Kaur, assessed the knowledge and attitude of nurses regarding stem cell and umbilical cord blood banking as the selected hospital of Ambala, Haryana, India. The objectives of the study were to assess the knowledge and attitude of nurses regarding stem cell and umbilical cord blood banking. Also to find out the correlation between knowledge and attitude score regarding stem cell and umbilical cord blood banking and to determine the association of level of knowledge and attitude of nurses regarding stem cell and umbilical cord blood banking. The Research approach adopted for the study was quantitative and it was carried out on the 170 nurses. A structured knowledge questionnaire and attitude scale (5 points Likert scale) was used to collect the data.

The result of the study depicts that more than half $(58 \%)$ of the nurses were in the age group of 20-25 years, majority of nurses (82\%) were having qualification in G.N.M, with majority of nurses $(89 \%)$ were in $1-5$ years of experience, More than half of nurses (52\%) having no additional information about umbilical cord blood banking required, more than half of the nurses $(51 \%)$ not heard about umbilical cord blood banking, majority of the nurses (94\%) having no training courses about umbilical cord blood sampling and stem cell sampling, forty three percent $(43 \%)$ of nurses had average knowledge, followed by $(28 \%)$ of nurses had good knowledge, $(19 \%)$ of nurses had very good knowledge and only (10\%)of nurses had excellent knowledge regarding stem cell umbilical cord blood banking, fifty one percent $(51 \%)$ of nurses had moderate favourable attitude regarding stem cells and umbilical cord blood banking and there was significant correlation between knowledge and attitude scores as evident by ' $r$ ' value of 0.15 . Validity was ensured in consultation with the guide and experts reliability of the knowledge questionnaire was tested by $k r 20(r=)$, for attitude scale by the Cronbach alpha method [16].

\section{Limitations}

The study was limited to:

- Short time duration of data collection

- Only Staff nurses present at the time of data collection. 


\section{Conclusion}

Hematopoietic stem cell transplantation (HSCT) is a potentially curative treatment for patients with blood disorders and genetic diseases. Stem cell transplantation (SCT) is a life-saving procedure for several malignant and non-malignant lifethreatening diseases. Nursing care of the patient undergoing a bone marrow transplant is complex and demands a high level of skill. Health care provider including nurses should have sound knowledge and understanding about stem cell transplantation.

There was a significant relationship found between educational package and knowledge score regarding haematopoietic stem cell transplantation. The obtained ' $t$ ' value of $15.80 *$ is higher than the table value, this indicates the effectiveness of the educational package. It was concluded educational package was effective in enhancing the knowledge of staff nurses.

\section{What does the study add to the existing knowledge?}

The nurse plays an important role in the informed consent process, supporting the medical staff's explanations and plans to ensure, as much as possible, that the patient is making an informed decision regarding HCT. Nurses play a key role in providing health care to patients and being close to the patients they can conduct projects and research studies in the hospitals. This type of studies emphasize the significance of the short term courses or in-service education for nurses in advanced knowledge on stem cell transplantation and making use of facilities available in the management of the patient in day to day activities.

\section{Recommendations}

Based on the finding of the study, the following recommendations are being made:

- A similar study can be conducted to assess the knowledge regarding stem cell and umbilical cord banking among staff nurses.

- A similar study can be conducted to assess the knowledge regarding cord blood collection technique among nursing student.

- A similar study can be conducted on a large sample size.
- A similar study can be conducted regarding the management of patients undergoing bone marrow transplantation.

- A similar study can be conducted to assess the knowledge regarding cord blood banking and stem cell therapy among antenatal women.

\section{Reference}

01. Lewis, Sharon \& Heitkemper Mclean.

[Crossref]

02. Margart, Driksen S O Brien, Bucher L. Medical surgical Nursing. (7th ed), Mosby- Elsevier. 2009;1006-1009.

[Crossref]

03. Jenq $R R$, van den Brink MR. Allogeneic haematopoietic stem cell transplantationindividualized stem cell and immune therapy of cancer. Nat Rev Cancer. 2010 Mar;10(3)213-21. doi: $10.1038 /$ nrc2804 [Crossref]

04. Al Hamed R, Bazarbachi AH, Malard F, Harousseau JL, Mohty M. Current status of autologous stem cell transplantation for multiple myeloma. Blood Cancer J. 2019 Apr 8;9(4)44.

doi: $\quad 10.1038 / s 41408-019-0205-9 \quad$ [Crossref]

05. Selvi TS. Stem cell therapy. International Journal of Advance in Nursing Management. 2017;5(4). [Crossref]

06. Bagilkar VV. Benefits of Stem Cells in Pediatrics. International Journal of Nursing Education and Research. 2017;5940;377-380.

[Crossref]

07. Muraro PA, Martin R, Mancardi GL, Nicholas R, Sormani MP, Saccardi R. Autologous haematopoietic stem cell transplantation for treatment of multiple sclerosis. Nat Rev Neurol. 2017 Jul;13(7)391-405.

doi: $10.1038 /$ nrneurol.2017.81 [Crossref]

08. Lee JY, Hong SH. Hematopoietic Stem Cells and Their Roles in Tissue Regeneration. Int J Stem Cells. $2020 ; 13(1) 1-12$. doi:10.15283/ijsc19127 [Crossref]

09. Michel Attal, Jean-Luc Harousseau, Thierry Facon, François Guilhot, et al. Single versus Double Autologous Stem-Cell Transplantation for Multiple. Myeloma J Med. 2003;349;2495-2502. DOI: $10.1056 /$ NEJMoa032290 [Crossref] 
10. Singh A K, McGuirk J P. Allogeneic stem cell transplantation- A historical and scientific overview. Cancer Research 2016;76(22)6445-6451. Doi: 10.1158/0008-5472.can-16-1311 [Crossref]

11. Hossam K, Mahmouda Alaa M, Elhaddadb Omar A, Fahmyc Mohamed A, Samraa Raafat M, Abdelfattaha Yasser $\mathrm{H}$, El-Nahassd Gamal M, Fathye Mohamed S. Abdelhadye Allogeneic hematopoietic stem cell transplantation for nonmalignant hematological disorders.

Doi: 10.1016/j.jare.2014.11.001 [Crossref]

12. Thakur Neha \& Timsy. Pre-experimental study to assess the effectiveness of structured teaching programme on knowledge regarding stem cell \& umbilical cord blood banking among staff nurses working at selected hospitals of Ludhiana, Punjab. Dec 2018;7(10)966-969.

[Crossref]

13. Bel G L, Abisha. Effectiveness of the Structures Teaching Programme on Stem Cell Therapy. IJANM. 2020;8(1).

DOI : $10.5958 / 2454-2652.2020 .00003 .7$ [Crossref]

14. Yesikar V, Banseria R, Dixit S, Shivram G. A cross- sectional study to assess the knowledge regarding stem cells and its transplantation among students from various colleges \& women from anc clinic in Indore. Public Health ReviewInternational Journal of Public Health Research. 2016;3(4)156-161.

[Article] [Crossref]
15. Praveena Narayanan, Alexandra Wolansky j Shawna, L Ehlers Mark R, Litzow Mrinal S, Patnaik William J, Hogan Shahrukh, K Hashmi. Medical Students' Knowledge, Familiarity, and Attitudes towards Hematopoietic Stem Cell Donation- Stem Cell Donation Behaviors. Biol Blood Marrow Transplant. 2016 Sep;22(9):1710-1716.

Doi: $\quad 10.1016 /$ j.bbmt.2016.06.014 [Crossref]

16. Anna M, Kamelska-Sadowska, Joanna Wojtkiewicz, Ireneusz M Kowalski. Review of the Current Knowledge on the Role of Stem Cell Transplantation in Neuro-rehabilitation. BioMed Research International. 2019.

[Article] [Crossref]

17. Neha Patyal, Ramanpreet Kaur, Ravnoorpreet Singh, Arushi, Reetu, Ridhim, Ritu, Rubeen Kaur, Sachin Kumar, Sarvjeet Kaur, Jyoti Sarin. A study to assess the knowledge and attitude of nurses regarding stem cell and umbilical cord blood banking in selected hospital of Ambala, Haryana. TPI. 2018;7(6)154-159.

[Crossref] 